\title{
Density functional theory study of $\mathrm{MnO}_{2}, \mathrm{TiO}_{2}$ and $\mathrm{VO}_{2}$
}

KP Maenetja, HR Chauke, PE Ngoepe

\author{
Materials Modelling Centre, University of Limpopo Private Bag X1106, Sovenga, 0727, South Africa \\ Email: khomotso.maenetja@gmail.com
}

\begin{abstract}
We investigate the structural stability of metal oxides $\beta-\mathrm{MnO}_{2}, \mathrm{TiO}_{2}$ and $\mathrm{VO}_{2}\left(\mathrm{MO}_{2}\right)$ which are used as catalyst in metal air batteries, using the density functional theory (DFT) within the generalized gradient approximation (GGA). Their mechanical property was determined to show the stability trend of the metal oxides catalyst. Cell parameters of the bulk structures of the $\mathrm{MO}_{2}$ are in reasonable agreement with the experimental values (deviations of approximately $0.8 \%$ and $-3.1 \%$ for $a$ and $c$, respectively, and of $1.6 \%$ in the cell volume). Phonon dispersion curves show that rutile ( $\mathrm{R}) \mathrm{TiO}_{2}$ is the most stable structure since it does not have vibrations in the negative frequencies.
\end{abstract}

\section{Introduction}

The Li-air battery has taken a lot of attention to researchers because of its ability to deliver ultra-high energy density and also gained its advantage because its lower weight due to the absence of metallic cathode.1 Metal oxides $\left(\mathrm{MO}_{2}\right)$ have attracted extensive attention because they possess various applications such as lithium- ion and Li-air batteries (LIBs), dye sensitized solar cells (DSSCs), fuel cells, catalysis, gas sensors, water splitting and super capacitors due to low cost and high compatibility with the environment. ${ }^{2}$

However, the fundamental challenges that limits the use of metal air battery technology is the ability to find a catalyst that will catalyse the formation and decomposition of $\mathrm{Li}_{2} \mathrm{O}_{2}$ during charging and discharging cycle i.e. oxygen reduction reaction (ORR) and oxygen evolution reaction (OER).

Catalytic materials have been proposed and synthesized to promote the oxygen reduction reactions and oxygen evolution reactions ORR/OER process, which can be mainly classified into three groups: carbon-based materials, ${ }^{3,4}$ noble metal/metal oxides ${ }^{5,6}$ and transition metal oxides. ${ }^{7,8}$ These catalytic materials show notable strengths and weaknesses, e.g. although noble metals/metal oxides, such as $\mathrm{Ru} / \mathrm{RuO}_{2},{ }^{9,10}$ could significantly decrease the ORR/ OER over-potentials, their high cost greatly hinders the practical application. In addition, some metal oxides have appeared to cause the decomposition of electrolytes. ${ }^{11}$

In this paper we investigate the electronic properties of the metal oxides which will give a better insight in approaching further calculations which will be applicable in the performance of the metal-air battery. The instability of the material does not rule out the ability of the material to be a better catalyst.

\section{Methodology}

The calculations were performed using ab initio density functional theory (DFT) formalism as implemented in the VASP total energy package ${ }^{12,13}$ with the projector augmented wave. ${ }^{14}$ The number of planewaves was determined by a cutoff kinetic energy of 500 $\mathrm{eV}$ and the Brillouin zone sampling scheme of Monkhorst-Pack with 6x6x9 k-points mesh for rutile $\mathrm{MnO}_{2}$ bulk structures. The phonon dispersion spectra were evaluated using PHONON code ${ }^{15}$ as implemented in the Materials Design within MedeA software of VASP code. ${ }^{16,17}$ The $2 \times 2 \times 3$ supercell was used for the tetragonal whereas the supercell of $2 \times 2 \times 2$ was used for monoclinic $\mathrm{MnO}_{2}$. Calculations carried out within the full Brillouin zone, parameters with interaction range of $7.0 \AA$, displacement of atoms was set at $0.02 \AA$ which gave a supercell which extends equally in all directions and does not reduce the symmetry of the system. The above parameters gave new lattice vectors of a', b' and c', $1 \times 2 \times 1$, 24 number of atoms in the supercell was 24 and resulted in 73 supercells to be calculated. Convergence was assumed when the maximum component of the residual forces on the ions was less than $0.01 \mathrm{eV} / \AA$.

\section{Results and discussion}

\subsection{Lattice parameters}

The lattice parameters allude to the physical dimension of unit cells in a crystal lattice. Lattices in three dimensions predominantly have three lattice constants, referred to as a, b, and c. However, the full set of lattice parameters consist of the three lattice constants and the three angles between them. Calculated lattice parameters are presents and the cell volume of the bulk structure of the metal oxide are shown in Table 1 and they are compared with the lattice parameters of the experimental lattice parameters calculated previously. ${ }^{18-20}$

Table 1: Lattice parameters and cell volume of tetragonal (rutile) $\mathrm{MnO}_{2}, \mathrm{TiO}_{2}$ and $\mathrm{VO}_{2}$ bulk structure

\begin{tabular}{llllll}
\hline Structure & \multicolumn{2}{c}{$\mathbf{a}(\AA \mathbf{A})$} & \multicolumn{2}{c}{$\mathbf{c}(\mathbf{\AA})$} & $\mathbf{V}\left(\mathbf{\AA}^{3}\right)$ \\
\hline$\beta-\mathrm{MnO}_{2}$ & 4.366 & $4.410[18]$ & 2.961 & $2.887[19]$ & 56.44 \\
$\beta-\mathrm{TiO}_{2}$ & 4.827 & $4.954[20]$ & 3.008 & $2.959[18]$ & 64.40 \\
$\beta-\mathrm{VO}_{2}$ & 4.617 & $4.554[18]$ & 2.774 & $2.857[19]$ & 59.13 \\
\hline
\end{tabular}

The lattice parameters were in good agreement with the experimental with deviations of approximately $+0.8 \%$ and $-3.1 \%$ for a and c, respectively, and of $1.6 \%$ in the cell volume for the $\mathrm{MO}_{2}$ shown in the above table. 


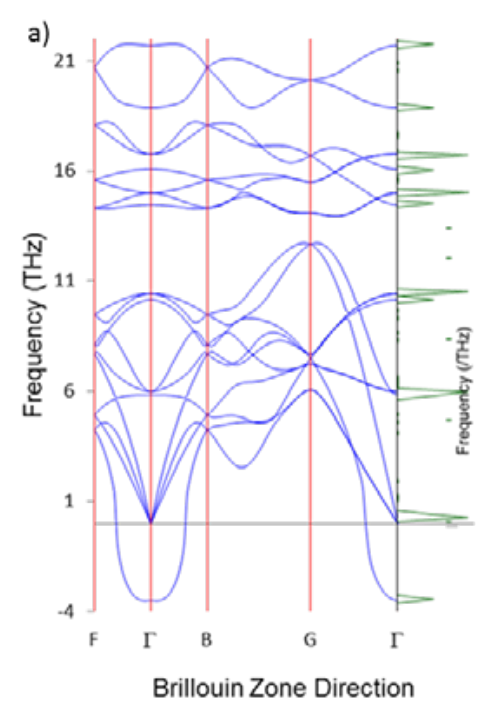

b) $\quad \mathrm{MnO}_{2}(\mathrm{R})$

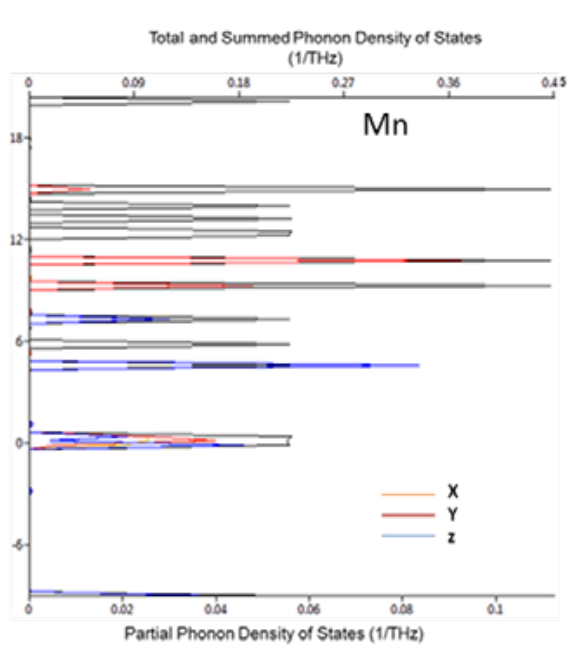

c)

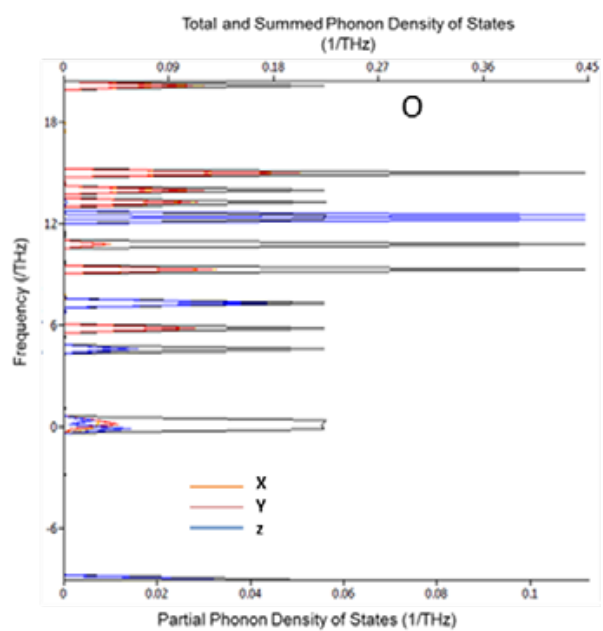

Figure 1: a) Phonon dispersion curve for $\mathrm{MnO}_{2}(\mathrm{R})$ b) Mn contribution and $\mathrm{O}$ contribution towards the vibrations along (x,y and $\left.\mathrm{z}\right)$ directions

a)

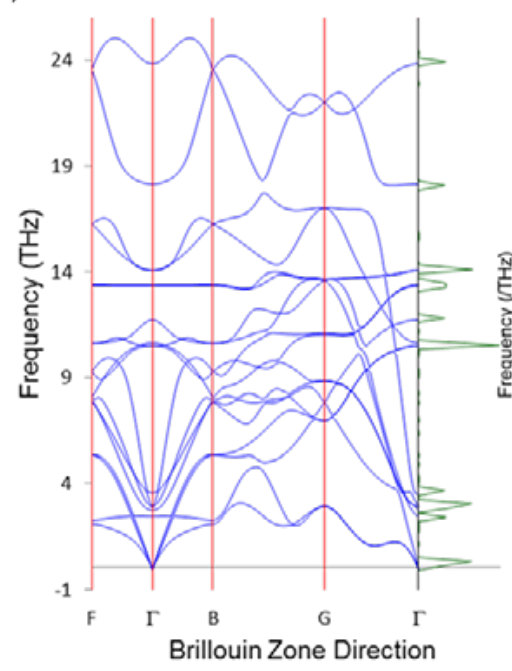

b)

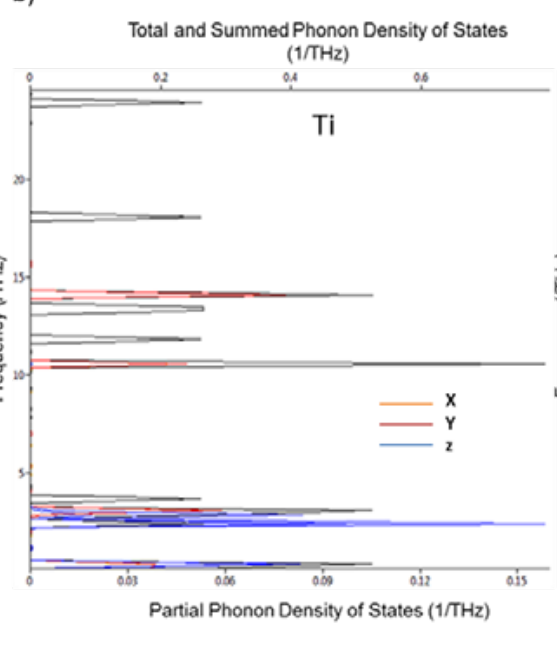

c)

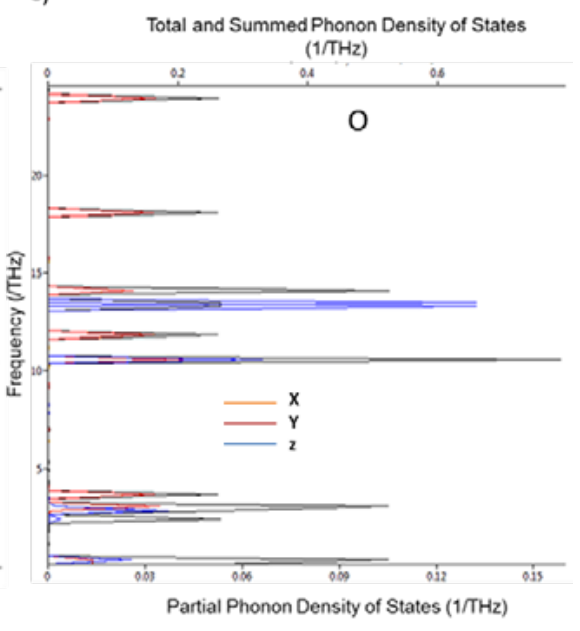

Figure 2: a) Phonon dispersion curve for $\left.\mathrm{TiO}_{2}(\mathrm{R}) \mathrm{b}\right)$ Ti contribution and $\mathrm{O}$ contribution towards the vibrations along (x, y and $\mathrm{z}$ ) directions

\subsection{Phonon dispersions}

The term phonon is used to draw an analogy between photon representing a quantum of electromagnetic radiation and quanta of lattice vibration. Theory of phonons explains most solid state phenomena, such as specific heat, melting, stability, thermal and electrical conductivity, etc., which cannot be explained with static lattice theory. ${ }^{21}$ In order to investigate the mechanical stability of the different structures of $\mathrm{MO}_{2}$, we have evaluated their phonon dispersion curves along symmetry direction within the first Brillouin zones and their elastic properties.

Rutile manganese dioxide structure with the phonon dispersion curve and PDOS shown in Figure 1 appeared to have negative frequencies along the $\Gamma$ Brillouin zone direction which extended to $-4 \mathrm{THz}$. The PDOS, however, it appears that both $\mathrm{Mn}$ and $\mathrm{O}$ are responsible for the negative frequencies (soft modes). The lower band of acoustic branches are associated with Mn atom while the upper band of the optical branches emanated from the $\mathrm{O}$ atom. The analysis of the phonon dispersion curve and PDOS associated to $\mathrm{TiO}_{2}(\mathrm{R})$ is shown in Figure 2; it is observed that there are no negative vibrations in all the Brillouin zone directions. The PDOS shows that the lower band of acoustic and upper optical bands emanated from $\mathrm{Ti}$ and $\mathrm{O}$ atoms. However, the lower bands of the acoustic branches were due to the contribution of Ti mostly along the $\mathrm{z}$ direction while the upper optical branches were due to $\mathrm{O}$ atom mainly along the $\mathrm{x}$ direction. The phonon dispersion curve and the PDOs for rutile $\mathrm{VO}_{2}$ which appeared to have negative frequencies along the $\Gamma, \mathrm{M}$ and $\mathrm{X}$ Brillouin zone direction shown in Figure 3. The negative frequencies extend to $-7 \mathrm{THz}$ in the $\Gamma$ Brillouin zone direction while for $\mathrm{M}$ and $\mathrm{X}$ direction the vibrations extend to -3THz. The PDOS showed that the lower band of acoustic and upper optical bands emanated from $\mathrm{V}$ and $\mathrm{O}$ atoms. The lower band of the acoustic branches were due to the contribution of $\mathrm{V}$ mostly along the $\mathrm{z}$ direction while the upper optical branches 


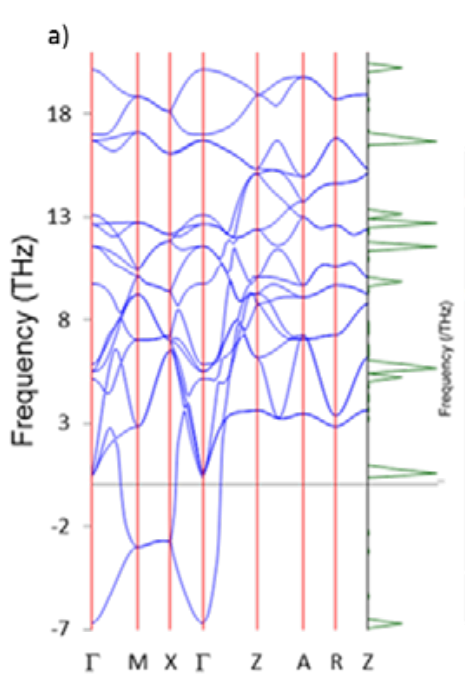

b) $\quad \mathrm{VO}_{2}(\mathrm{R})$

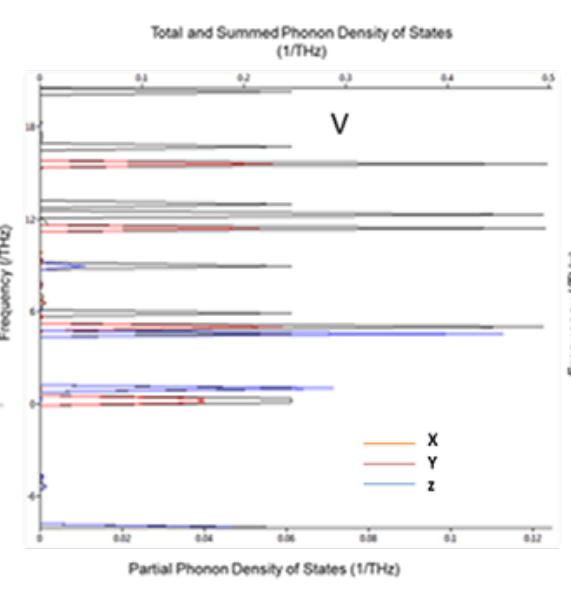

c)

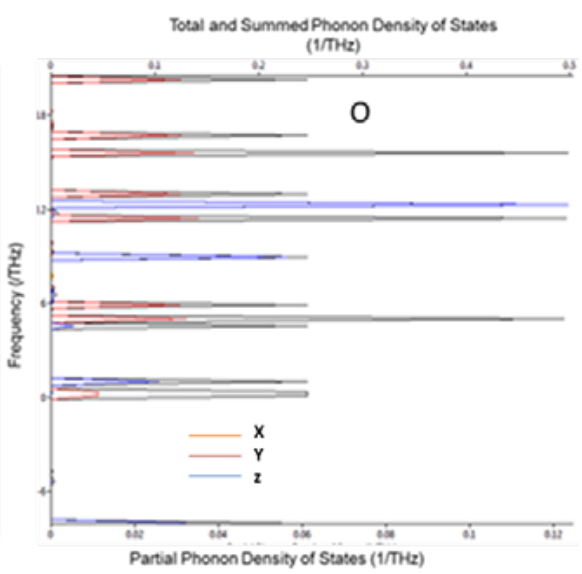

Brillouin Zone Direction

Figure 3: a) Phonon dispersion curve for $\mathrm{VO}_{2}(\mathrm{R})$ b) Ti contribution and $\mathrm{O}$ contribution towards the vibrations along (x, y and $\mathrm{z}$ ) directions

were due to $\mathrm{O}$ atom mainly along the $\mathrm{x}$ and $\mathrm{y}$ direction and a little contribution of $\mathrm{V}$ atom along $\mathrm{x}$ direction.

\section{Conclusions}

Mechanical stability study of the metal oxide $\left(\mathrm{MnO}_{2}, \mathrm{TiO}_{2}\right.$ and $\mathrm{VO}_{2}$ ) were successfully carried out using ab-initio plane wave pseudo potential method. The phonon dispersion curves showed that rutile $\mathrm{MnO}_{2}$ and $\mathrm{VO}_{2}$ structures were unstable because they had negative frequencies (soft modes) while rutile $\mathrm{TiO}_{2}$ showed that the structure is stable since phonon dispersion curves do not have soft modes.

\section{Acknowledgements}

National Research Foundation for financial assistance and South African Research Chair Initiative of the Department of Science and Technology. The calculations performed were carried out at the Centre for High Performance Computing in Cape Town, South Africa; some were performed at our local clusters at Materials Modelling Centre, University of Limpopo, South Africa.

\section{References}

1. K. M. Abraham, C. O. (2010). The Electrochemical Society 217th Meeting Abs. 0745, 1.

2. http://wiesner.mse.cornell.edu/res_energy.htm 11/11/2015. (n.d.).

3. J. Yi, K. Liao, C. Zhang, T. Zhang, F. Li and H. Zhou. (2015). Facile in situ preparation of graphitic- $\mathrm{C}_{3} \mathrm{~N}_{4} @$ carbon aaper as an efficient metal-free cathode for nonaqueous $\mathrm{Li}-\mathrm{O}_{2}$ battery, ACS Applied Material. Interface, 7, 10823.

4. W. Zhou, H. Zhang, H. Nie, Y. Ma, Y. Zhang and H. Zhang (2015). Hierarchical micron-sized mesoporous/macroporous graphene with well-tuned surface oxygen chemistry for high capacity and cycling stability Li- $\mathrm{O}_{2}$ battery, ACS Applied. Material. Interfaces, 7, 3389.

5. M. Lee, Y. Hwang, K. Yun and Y. Chung. (2015). Greatly improved electrochemical performance of lithium-oxygen batteries with a bimetallic platinum-copper alloy catalyst, Journal of Power Sources, 288, 296.

6. K. Liao, T. Zhang, Y. Wang, F. Li, Z. Jian, H. Yu and H. Zhou. (2015). Nanoporous $\mathrm{Ru}$ as a carbon- and binder-free cathode for
$\mathrm{Li}-\mathrm{O}_{2}$ batteries, Journal of chemistry and sustainability, energy and materials $8,1429$.

7. S. Jee, W. Choi, C.H. Ahn, G. Yang, H.K. Cho, J. Lee and C. Yu. (2015). Enhanced oxygen reduction and evolution by in situ decoration of hematite nanoparticles on carbon nanotube cathodes for high-capacity nonaqueous lithium-oxygen batteries, Journal of Materials. Chemistry A, 3, 13767.

8. F. Li, D. Tang, Y. Chen, D. Golberg, H. Kitaura, T. Zhang, A. Yamada and H. Zhou. (2013). Ru/ITO: A carbon-free cathode for nonaqueous $\mathrm{Li}_{-}$, batteries, Nano Letters, 13, 4702.

9. K. Liao, X. Wang, Y. Sun, D. Tang, M. Han, P. He, X. Jiang, T. Zhang and H. Zhou. (2015) An oxygen cathode with stable full discharge-charge capability based on 2D conducting oxide, Energy Environmental Science, 8, 1992.

10. F. Li, D. Tang, Z. Jian, D. Liu, D. Golberg, A. Yamada and H. Zhou. (2014). Li- $\mathrm{O}_{2}$ battery based on highly efficient $\mathrm{Sb}$-doped tin oxide supported Ru nanoparticles, Advanced Materials, 26, 4659.

11. B.D. McCloskey, R. Scheffler, A. Speidel, D.S. Bethune, R.M Shelby and A.C. Luntz. (2011). On the efficacy of electrocatalysis in nonaqueous $\mathrm{Li}-\mathrm{O}_{2}$ batteries, Journal of the American Chemical Society, 133, 18038.

12. G. Kresse and J. Hafner. (1994). Theory of the crystal structures of selenium and tellurium: the effect of generalized-gradient corrections to the local-density approximation, Physical Review B, 49, 1425.

13. J. P. Perdew, K. B. (1996). Generalized gradient approximation made simple, Physical Review Letters, 77, 3859.

14. G. Kresse and D. Joubert. (1999). From ultrasoft pseudopotentials to the projector augmented-wave method, Physical Review B, 59, 1758.

15. K. Parlinski, Li ZQ, Y. Kawazoe. (1997). First-Principles determination of the soft mode in cubic $\mathrm{ZrO}_{2}$ Physical Review Letters, $78,4063$.

16. G. Kresse and J. Futhmuller (1996). Efficient iterative schemes for ab-initio total-energy calculations using a plane-wave basis set, Physical Review, 54, 1169.

17. G. Kresse and J. Furthmuller (1996). Efficiency of ab-initio total energy calculations for metals and semiconductors using a plane-wave basis set, Computational. Materials Science, 6, 15.

18. K. D. Rogers. (1993). An X-ray diffraction study of semiconductor and metallic vanadium dioxide, Powder Diffraction, 8, 240.

19. T. Hahn. (1989). International tables of crystallography, volume A space group symmetry (Vol. 2nd revised edition).

20. S. C. Abrahams and J. L. Bernstein. (1971). Rutile: normal probability plot analysis and accurate measurement of crystal structure, The Journal of Chemical Physics, 55, 3206.

21. Chen, H. (1996). Metallurgy Industry Press. Beijing. 